\title{
Phylogeny of Vibrio vulnificus from the Analysis of the Core-Genome: Implications for Intra-Species Taxonomy
}

\author{
Francisco J. Roig ${ }^{1,2,3}$, Fernando González-Candelas ${ }^{4,5}$, Eva Sanjuán ${ }^{1,2}$, Belén Fouz ${ }^{1,2}$, \\ Edward J. Feil ${ }^{6}$, Carlos Llorens ${ }^{3}$, Craig Baker-Austin ${ }^{7}$, James D. Oliver ${ }^{8,9}$, \\ Yael Danin-Poleg ${ }^{10}$, Cynthia J. Gibas ${ }^{11}$, Yechezkel Kashi ${ }^{10}$, Paul A. Gulig ${ }^{12}$, \\ Shatavia S. Morrison ${ }^{11}$ and Carmen Amaro ${ }^{1,2 *}$
}

${ }^{1}$ Estructura de Investigación Interdisciplinar en Biotecnología y Biomedicina BIOTECMED, University of Valencia, Valencia, Spain, ${ }^{2}$ Departmento de Microbiología y Ecología, Universidad de Valencia, Valencia, Spain, ${ }^{3}$ Biotechvana, Parc Cientific, Universitat de Valencia, Valencia, Spain, ${ }^{4}$ Joint Research Unit on Infection and Public Health FISABIO-Salud Pública and Universitat de Valencia-I2SysBio, Valencia, Spain, ${ }^{5}$ CIBEResp, National Network Center for Research on Epidemiology and Public Health, Instituto de Salud Carlos III, Valencia, Spain, ${ }^{6}$ Department of Biology and Biochemistry, University of Bath,

OPEN ACCESS

Edited by:

Jesus L. Romalde,

Universidade de Santiago de Compostela, Spain

Reviewed by:

Javier Pascual,

German Collection of Microorganisms and Cell Cultures (LG), Germany Karla Satchell,

Feinberg School of Medicine, Northwestern University, United States

*Correspondence: Carmen Amaro carmen.amaro@uv.es

Specialty section

This article was submitted to Evolutionary and Genomic Microbiology,

a section of the journal

Frontiers in Microbiology

Received: 13 October 2017 Accepted: 14 December 2017

Published: 05 January 2018

Citation:

Roig FJ, González-Candelas F, Sanjuán E, Fouz B, Feil EJ, Llorens C,

Baker-Austin C, Oliver JD,

Danin-Poleg Y, Gibas CJ, Kashi Y, Gulig PA, Morrison SS and Amaro C (2018) Phylogeny of Vibrio vulnificus from the Analysis of the

Core-Genome: Implications for Intra-Species Taxonomy.

Front. Microbiol. 8:2613.

doi: 10.3389/fmicb.2017.02613
Bath, United Kingdom, ${ }^{7}$ Centre for Environment, Fisheries and Aquaculture Science, Weymouth, United Kingdom, ${ }^{8}$ Department of Biological Sciences, University of North Carolina at Charlotte, Charlotte, NC, United States, ${ }^{9}$ Duke University Marine Lab, Beaufort, NC, United States, ${ }^{10}$ Faculty of Biotechnology and Food Engineering, Technion-Israel Institute of Technology, Haifa, Israel, ${ }^{11}$ Department of Bioinformatics and Genomics, the University of North Carolina at Charlotte, Charlotte, NC, United States, ${ }^{12}$ Department of Molecular Genetics and Microbiology, University of Florida, Gainesville, FL, United States

Vibrio vulnificus $(\mathrm{Vv})$ is a multi-host pathogenic species currently subdivided into three biotypes (Bts). The three Bts are human-pathogens, but only Bt2 is also a fish-pathogen, an ability that is conferred by a transferable virulence-plasmid (pVvbt2). Here we present a phylogenomic analysis from the core genome of $80 \mathrm{~V} v$ strains belonging to the three Bts recovered from a wide range of geographical and ecological sources. We have identified five well-supported phylogenetic groups or lineages (L). L1 comprises a mixture of clinical and environmental Bt1 strains, most of them involved in human clinical cases related to raw seafood ingestion. $\mathrm{L} 2$ is formed by a mixture of Bt1 and Bt2 strains from various sources, including diseased fish, and is related to the aquaculture industry. L3 is also linked to the aquaculture industry and includes Bt3 strains exclusively, mostly related to wound infections or secondary septicemia after farmed-fish handling. Lastly, L4 and L5 include a few strains of Bt1 associated with specific geographical areas. The phylogenetic trees for Chrl and II are not congruent to one another, which suggests that inter- and/or intra-chromosomal rearrangements have been produced along $\mathrm{V} v$ evolution. Further, the phylogenetic trees for each chromosome and the virulence plasmid were also not congruent, which also suggests that pVvbt2 has been acquired independently by different clones, probably in fish farms. From all these clones, the one with zoonotic capabilities (Bt2-Serovar E) has successfully spread worldwide. Based on these results, we propose a new updated classification of the species based on phylogenetic lineages rather than on Bts, as well as the inclusion of all Bt2 strains in a pathovar with the particular ability to cause fish vibriosis, for which we suggest the name "piscis."

Keywords: microbial evolution, pathogens, SNP, Vibrio vulnificus, core genome, virulence plasmid, pathovar, biotype 


\section{INTRODUCTION}

Vibrio vulnificus is an emerging zoonotic pathogen that inhabits brackish water ecosystems from temperate and tropical areas and whose geographical distribution has recently extended to Northern countries due to global warming (Baker-Austin et al., $2013,2017)$. The pathogen survives in water, either associated with the mucous surfaces of algae and aquatic animals or as a free-living bacterium that can be concentrated by filtering organisms such as oysters (Oliver, 2015).

V. vulnificus was defined as a bacterial species in 1976 (Farmer, 1979) and was later split into three biotypes (Bt) on the basis of differences in genotypic and phenotypic traits as well as in host range (Tison et al., 1982; Bisharat et al., 1999). The three Bts are opportunistic human pathogens, but Bt 2 is also pathogenic for aquatic animals. The zoonotic strains belong to the same serovar (Ser) and are classified as Bt2-SerE (Biosca et al., 1997).

The various diseases caused by this species are known as vibriosis. Human vibriosis presents as two main forms depending on the pathogen's route of entry into the body, ingestion or contact (Oliver, 2015). In the first case, the pathogen is ingested with raw seafood, colonizes the intestine and causes gastroenteritis and/or primary septicemia. In the second case, the pathogen crosses the skin barrier during an injury or directly colonizes a preexisting wound causing local but severe necrosis and/or secondary septicemia. The main factor that predisposes to death by sepsis is a high level of serum iron as a consequence of multiple pathologies (e.g., hemochromatosis, diabetes, cirrhosis, and viral hepatitis) (Oliver, 2015).

Epidemiological data on human vibriosis from the Centers for Disease Control and Prevention (CDC) estimate that around 80,000 people are infected by Vibrio spp. each year in the USA and that, of these, V. vulnificus is responsible for most of the fatal cases (case fatality rate $>50 \%$ for septicemia; Jones and Oliver, 2009). Thus, V. vulnificus is responsible for over $95 \%$ of seafood-related deaths (Jones and Oliver, 2009), the highest fatality rate of any known food-borne pathogen (Rippey, 1994). In addition, increasing incidents of infections are occurring globally, with cases reported in Europe and Asia (Hlady and Klontz, 1996; Baker-Austin et al., 2013; Lee et al., 2013b). Crucially, infections currently appear to be increasing in both the USA and in Europe (Newton et al., 2012; Baker-Austin et al., 2013).

Regarding fish vibriosis, eels seem to be the most susceptible host, especially under farming conditions (Amaro et al., 2015). The pathogen colonizes the eel gills, enters the blood and causes death by septicemia, even in healthy individuals (Marco-Noales et al., 2001). Eel vibriosis occurs in farms as epizootics or outbreaks of high mortality that can lead to the closure of the farm if the disease is not controlled promptly. Epizootiological data suggest that outbreaks of eel vibriosis have been registered in all of the countries where eels are cultured in brackish waters, including fish farms located in Northern-European countries (Haenen et al., 2013).

The genetic basis for human virulence is only partially known, although most studies suggest that all strains of the species may have the ability to infect humans regardless of their origin, clinical, or environmental (Gulig et al., 2005). In contrast, the ability to infect fish is dependent on a virulence plasmid (pVvBt2) that is only present in Bt2 strains (Lee et al., 2008; Roig and Amaro, 2009). The plasmid encodes resistance to the innate immunity of eels, and probably other teleost (Lee et al., 2008, 2013a; Pajuelo et al., 2015). Interestingly, pVvbt2 can be transmitted among bacteria aided by a second, conjugative plasmid, which is widespread in the species (Lee et al., 2008).

The aim of this study is to describe the phylogenetic groups within the species and compare them with the current intraspecific groups from the characterization of the core genome of species (CGS), the core genome of plasmid pVvbt2 (CGP) and the core genome of human virulence-related genes (CGV). Our results highlight the importance of aquaculture industry in the recent evolution and epidemic spread of the species and support the intra-specific classification in lineages instead of Bts, as well as the inclusion of a pathovar grouping all fish virulent isolates for which we propose the name "piscis."

\section{MATERIALS AND METHODS}

\section{Bacterial Isolates, Culture Conditions, DNA Extraction, and Sequencing}

The genomes used in this study and the main features of the corresponding strains are detailed in Table 1. The strains whose genome was sequenced are marked in Table 1. These strains were routinely grown in Tryptone Soy Broth or agar plus 5 $\mathrm{g} / \mathrm{l} \mathrm{NaCl}$ (TSB-1 or TSA-1, Pronadisa, Spain) at $28^{\circ} \mathrm{C}$ for $24 \mathrm{~h}$. The strains were maintained both as lyophilized stocks and as frozen stocks at $-80^{\circ} \mathrm{C}$ in marine broth (Difco) plus $20 \%(\mathrm{v} / \mathrm{v}$ ) glycerol.

DNA was extracted using GenElute ${ }^{\mathrm{TM}}$ Bacterial Genomic DNA (Sigma, Spain) from bacteria grown with shaking at $28^{\circ} \mathrm{C}$ for $12 \mathrm{~h}$. Samples with a DNA concentration of $10-15 \mathrm{ng} / \mu \mathrm{l}$ were used for sequencing with Illumina Genome Analyzer technology GAII (Illumina MiSeq) flow cell in the Genome Analysis Centre in Norwich (UK) and the SCSIE of the University of Valencia (Spain). To this end, unique index-tagged libraries for each sample (up to 96 strains) were created using TruSeq DNA Sample Preparation for subsequent cluster generation (Illumina cBot), and up to 12 separate libraries were sequenced in each of eight channels in Illumina Genome Analyser GAII cells with 100base paired-end reads. The index-tag sequence information was used for downstream processing to assign reads to the individual samples (Harris et al., 2010).

\section{Genomes Selected as Reference}

The genomes of the Bt1 strain YJ016 (NC_005139 and NC_005140) and the plasmids pR99 (AM293858) and p4602-2 (AM293860) were used as templates for all the genomic analysis. The genome of the strain YJ016 was selected because it is one of the few genomes of $V$. vulnificus that has been accurately closed and annotated (Chen et al., 2003). The YJ016 genome contains 5,097 genes (3,387 in chromosome I and 1,710 in chromosome II), pR99 contains 71 genes and p4602-2 contains 67 genes. 
TABLE 1 | Origin, year of isolation, biotype, serovar, virulence-related typing, and genome accession number of $V$. vulnificus strains used in this study.

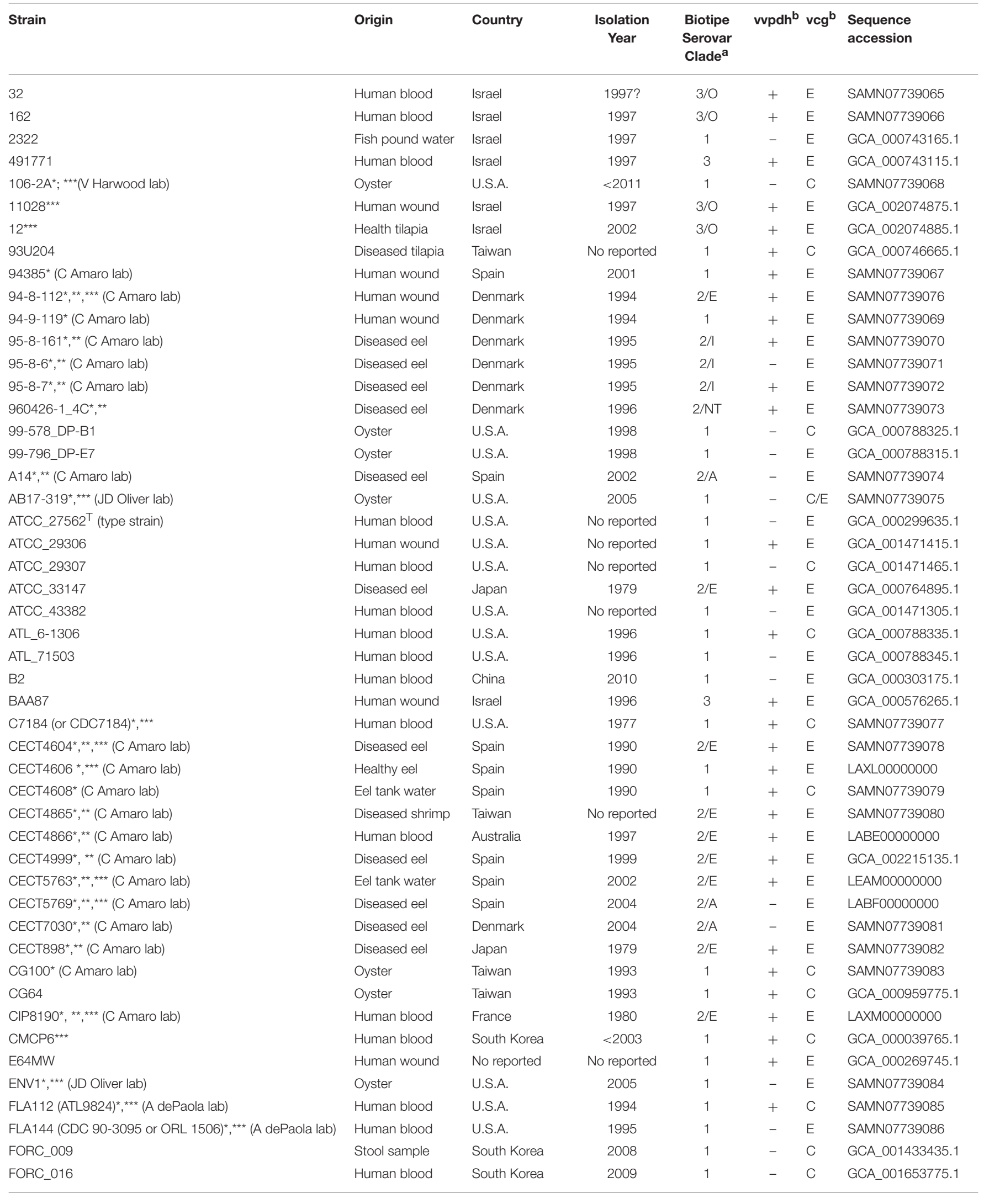


TABLE 1 | Continued

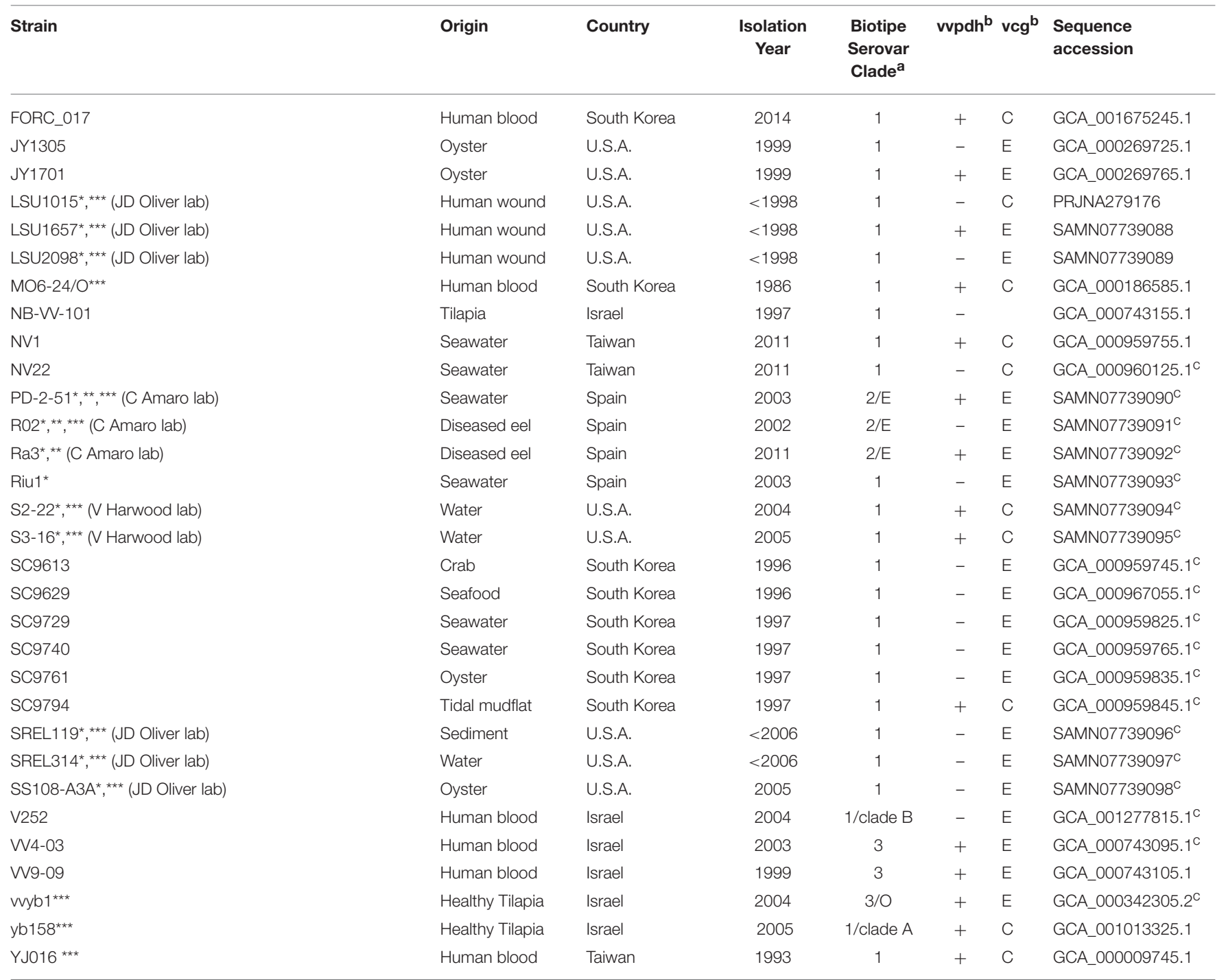

${ }^{*}$ Strains whose genomes were sequenced in this study. The laboratory that purchased the strain is indicated in parenthesis. ${ }^{* \star}$ Strains used for virulence plasmid analysis. ${ }^{\star \star \star}$ Strains used for Vibrio species analysis.

${ }^{a}$ O-antigen serovar was determined for Bt2 and 3 isolates according to Biosca et al. (1997): Clade A and B were described by Danin-Poleg et al. (2015) and Efimov et al. (2015), respectively. NT, non typable.

${ }^{b}$ vvpdh (V. vulnificus potentially dangerous for humans); pilF polymorphism associated with human virulence (Roig et al., 2010): vcg (virulence correlated gene: E, environmental type, C, clinical type, C/E, clinical and environmental type) (Rosche et al., 2005).

${ }^{c}$ Waiting for definitive accession.

\section{Genome Sequence Assembly}

Reads for each genome were done using SPAdes version 3.6.1 (Bankevich et al., 2012) with kmers from 21 to 127 and the careful option to reduce mismatches and short indels. Then, multiple sequence alignments were obtained by using Progressive Mauve software with default options (Darling et al., 2004). Locally Collinear Blocks (LCBs) with a size larger than $1 \mathrm{~kb}$ that were present in all the genomes were used to define the core genomes. The selected LCBs were concatenated to be used in subsequent analyses to build a core genome multiple alignment.

\section{Core Genome}

We selected seven Vibrio species that had at least one fully sequenced and annotated genome to define the core genome of the genus (CGG). Table $\mathbf{S 1}$ summarizes the characteristics of the closed Vibrio genomes selected for the study.

The identification of each gene was performed using local BLAST searches (Wang et al., 2003). Three independent searches, one per chromosome and plasmid, were performed, and a database was generated for each genome. The resulting sequences were mapped onto reference genes using Geneious 6.1.6 (Biomatters) software. As the sequences used for the 
generation of the databases were not closed genomes (draft genomes/contigs), only the genes present in all the genomes, with a minimum length of $80 \%$ of the total length of the homologous gene in the reference genome and with a DNA identity higher than $70 \%$, were included in the CGS, the CGP or the CGG for further analysis.

In order to refine the preliminary alignment for the entire core, individual alignments for each gene were performed using the program MAFFT (Katoh and Standley, 2013). The elimination of unaligned ends and genes that did not match the above requirements was performed using an in-house Python script. Once all the genes were aligned, a concatenated sequence was generated for the CGS, the CGP and the CGG.

\section{Functional Analysis of the CGS}

Functional annotation was performed using GPRO (Futami et al., 2011). Gene descriptions were obtained by blasting the predicted protein sequences against the NCBI non-redundant proteins (NR) and the Clusters of Orthologous Groups (COGs) databases (Tatusov, 2000) as reference subjects. Protein accessions obtained from the annotation based on NR were used to add additional Gene Ontology (GO) (Gene Ontology Consortium, 2008) and Enzyme Commission (EC) (Bairoch, 2000) designations. Information about metabolic pathways was retrieved via the web from the KEGG database (Kotera et al., 2012) based on EC numbers relation.

\section{Single Nucleotide Polymorphisms (SNPs) Identification}

SNPs were identified in the genomes from the coding regions as described previously (Harris et al., 2010) with appropriate SNP cutoffs to minimize the number of false-positive/negative calls. SNPs were filtered to remove those at sites with a SNP quality score lower than 40 and/or with read coverage below $25 \mathrm{X}$ in this region. SNPs at sites with heterogeneous mappings were also filtered out if the SNP was present in fewer than $85 \%$ of reads for that position.

\section{Virulence Genes to Define the Human Virulence-Related Core Genome (VCGS)}

We identified human virulence-related genes in the CGS from previously published data to define the human virulence-related core genome (VCGS) (Chakrabarti et al., 1999; Chen et al., 2004; Horstman et al., 2004; Gulig et al., 2005; Bogard and Oliver, 2007; Alice et al., 2008; Oh et al., 2008; Brown and Gulig, 2009; Liu et al., 2009; Lee et al., 2010; Chen and Chung, 2011; Kim et al., 2011).

\section{Phylogenetic Reconstruction and Congruence Analysis}

All the phylogenetic trees for CGG, CGS, CGP, and VCGS were reconstructed using the maximum-likelihood (ML) method with PhyML software (Guindon et al., 2009). The best evolutionary model for each dataset was determined with jModelTest (Posada, 2008) and considering the Akaike information criterion (AIC) (Akaike, 1974). The selected models were the generalized timereversible model $(\mathrm{GTR})$ with Gamma $(+\mathrm{G})$ distribution and invariant positions (+I) (Tavaré, 1986) for SNP analysis. The pVvBt2 phylogeny was evaluated using the Hasegawa-KishinoYano model (Hasegawa et al., 1985). Support for the nodes derived in these reconstructions was evaluated by bootstrapping using 1,000 replicates (Felsenstein, 1985).

The congruence among the different phylogenetic reconstructions was evaluated using Shimodaira-Hasegawa (SH) (Shimodaira and Hasegawa, 1999) and expected-likelihood weight (ELW) tests as implemented in TreePuzzle version 5.2 (Schmidt et al., 2002; Strimmer and Rambaut, 2002).

\section{RESULTS}

\section{Core genome}

We obtained almost complete genome sequences of $38 \mathrm{~V}$. vulnificus strains (Table 1). The CGS was inferred from these genomes and additional genomes taken from the database $(80$ in total). The analysis included strains of the three Bts, isolated from a variety of hosts and habitats all over the world (Table 1). The main characteristics of the analyzed genomes and the CGS as well as the number of SNPs identified per chromosome are detailed in Tables 2, 3, respectively. The average gene identities in the CGS were 91 and $89 \%$ for chromosome I and II, respectively, which supports previous observations pertaining to the greater variability of chromosome II in Vibrio spp. (Kirkup et al., 2010).

The genes of the CGS and the associated metabolic pathways are shown in Tables S2, S3, respectively. The genes present in all the strains that did not match the criteria used to define the CGS (spanning less than $80 \%$ of the length and showing less than $70 \%$ identity with respect to the homologous gene in the reference YJ016 genome) are shown in Table S4. The CGS includes practically all of the genes for glycolysis, TCA cycle and pentose phosphate pathway, aerobic and anaerobic respiration, nitrate respiration, as well as for biosynthesis of metabolic intermediates, cofactors, nucleotides, amino acids and cell building blocks (Tables S2, S3). The CGS also includes genes involved in survival in different environments (water, animal intestine, chitinous surfaces), such as genes for resistance to different stressors (e.g., cold, heat, toxic oxygen forms, antimicrobials, and tellurite), genes for chitin degradation (i.e., various chitinases) and genes for surface colonization [e.g., pilus MSHA (mannose sensitive hemagglutination) and flagellum biogenesis]. It is generally assumed that genes on the Vibrio chromosome II have specific environmental functions related to habitat adaptation (Xu et al., 2003).

TABLE 2 | Some general data of the genome and core genome of the species (CGS) V. vulnificus

\begin{tabular}{lccccc}
\hline & $\begin{array}{c}\text { Average Genome size } \\
\text { in bp (number of } \\
\end{array}$ & \multicolumn{4}{c}{ Core } \\
\cline { 3 - 6 } & genes) & Size (bp) & Genes & SNP & $\%$ G+C \\
\hline Chromosome I & $3,286,174(3,103)$ & $1,396,961$ & 1,304 & 132,027 & 46.9 \\
Chromosome II & $1,803,986(1,571)$ & 519,724 & 414 & 45,027 & 46.8 \\
Chromosome I+ II & $5,090,160(4,674)$ & $1,916,685$ & 1,718 & 177,054 & 46.9
\end{tabular}


TABLE 3 | Number of SNPs sites per lineages identified in the core genome of the species (CGS).

\begin{tabular}{|c|c|c|c|c|c|c|}
\hline \multirow[t]{2}{*}{ Lineage } & \multicolumn{3}{|c|}{ Chromosome I } & \multicolumn{3}{|c|}{ Chromosome II } \\
\hline & Specific SNPs & $\begin{array}{c}\% \text { Specific SNPs of } \\
\text { total SNPs }\end{array}$ & $\begin{array}{l}\text { \% Specific SNPs of } \\
\text { Core Genome }\end{array}$ & Specific SNPs & $\begin{array}{c}\% \text { Specific SNPs of } \\
\text { total SNPs }\end{array}$ & $\begin{array}{c}\% \text { Specific SNPs of } \\
\text { Core Genome }\end{array}$ \\
\hline L1 & 83,107 & 62.95 & 5.95 & 28,193 & 62.61 & 5.42 \\
\hline L2 & 63,308 & 47.95 & 4.53 & 21,870 & 48.57 & 4.21 \\
\hline L3 & 25,034 & 18.96 & 1.79 & 9,619 & 21.36 & 1.85 \\
\hline Ł4 & 15,989 & 12.11 & 1.14 & 6,090 & 13.53 & 1.17 \\
\hline L5 & 132,027 & 100 & 9.45 & 45,027 & 100 & 8.66 \\
\hline L1-L2 & 117,305 & 88.85 & 8.4 & 39,924 & 88.67 & 7.68 \\
\hline L1-L3 & 91,163 & 69.05 & 6.53 & 30,933 & 68.7 & 5.95 \\
\hline L1-L4 & 89,724 & 67.96 & 6.42 & 30,230 & 67.14 & 5.82 \\
\hline L1-L5 & 87,874 & 66.56 & 6.29 & 29,778 & 66.13 & 5.73 \\
\hline L2-L3 & 74,594 & 56.5 & 5.34 & 26,214 & 58.22 & 5.04 \\
\hline L2-L4 & 85,808 & 64.99 & 6.14 & 29,097 & 64.62 & 5.6 \\
\hline L2-L5 & 71,527 & 54.18 & 5.12 & 24,968 & 55.45 & 4.8 \\
\hline L3-L4 & 52,708 & 39.92 & 3.77 & 18,246 & 40.52 & 3.51 \\
\hline L3-L5 & 34,277 & 25.96 & 2.45 & 12,514 & 27.79 & 2.41 \\
\hline L4-L5 & 42,081 & 31.87 & 3.01 & 13,676 & 30.37 & 2.63 \\
\hline
\end{tabular}

Regarding the metabolic pathways, all of the common genes for general metabolic pathways such as butanoate metabolism, glycerolipid metabolism, peptidoglycan biosynthesis and pyrimidine metabolism are located on chromosome I while the genes for biosynthesis of siderophores, which could be related to habitat adaptation, are in the chromosome II but together with other metabolic genes not clearly related to habitat adaptation (i.e., fatty acid biosynthesis/elongation, glycerophospolipid metabolism, glyoxylate/dicarboxylate metabolism, nicotinate/nicotinamide metabolism, porphyrin metabolism, and valine/leucine/isoleucine metabolism) (Table S3). Regarding to survival genes, genes for the flagellum and MSHA pilus are located in chromosome I while those for the Flp/Tad pilus are located in chromosomes I and II (Table S2). In conclusion, there is no clear relationship between chromosome localization and habitat adaptation for the CGS-genes.

Figure 1 presents the distribution of gene ontology terms. Most of the genes in the CGS encode proteins associated with cell membranes that are related with regulation of transcription, transport or metabolic/oxido-reduction process and present catalytic/hydrolase, transferase or transcription-factor activity (Figure 1). Again, many functions associated to CGS genes in chromosome I were not found in chromosome II such as kinase activity, metal ion transport and phospho-relay response regulator activity, etc. (Figure 1). In addition, no CGS genes related to proteolysis, phospho-relay signal transduction, carbohydrate transport, phosphorylation, ATP catabolic process, chemotaxis, biosynthesis, DNA recombination and repair, and phosphoenolpyruvate-dependent sugar phosphotransferase system were identified in chromosome II (Figure 1).

To determine the CGP, the plasmid was reconstructed from the sequenced genomes of the Bt2 strains indicated in Table 1.
According to the phylogenetic tree (Figure 3), we found six variants of the plasmid, two previously described (types II and IV) (Lee et al., 2008; Roig and Amaro, 2009) and four new ones (Table 4). The list of genes in the CGP is shown in Table S5. The CGP includes three virulence genes, two host-specific, vep07 and $f t b p$ (fish transferrin binding protein), involved in resistance to and growth in eel serum, respectively (unpublished results, Pajuelo et al., 2015), and one host-nonspecific, $r t x A 1$. This is a mosaic gene related with resistance to phagocytosis by murine and eel phagocytes (Lee et al., 2013a; Satchell, 2015) that presents at least seven different forms in V. vulnificus (Roig et al., 2011; Satchell, 2015).

\section{Phylogenomic Analysis}

To root the V. vulnificus phylogenetic tree within the genus, we first reconstructed a phylogenetic tree from the closed genomes of 15 strains belonging to seven Vibrio species ( $V$. parahaemolyticus, V. anguillarum, V. algynolyticus, V. campbellii, $V$. furnissii, V. cholerae, and V. splendidus-clade) (main genome characteristics shown in Table S1) together with 27 selected V. vulnificus genomes (Table 1). Maximum-likelihood (ML) trees for chromosome I, II and I+II were reconstructed based on the common genes of these Vibrio spp. (Figure S1). The ML trees showed $V$. vulnificus as a very compact and independent group. Next, we inferred the phylogeny of the species from the $80 \mathrm{~V}$. vulnificus genomes indicated in Table 1 by using ML reconstruction obtained from the SNPs of coding regions.

The phylogenetic trees for $V$. vulnificus are shown in Figure 2 and Figure S2. The rooted V. vulnificus phylogenetic reconstructions for chromosomes I and II were compared by congruence tests (Table 5). The results clearly indicate that chromosomes I and II were not congruent with one 


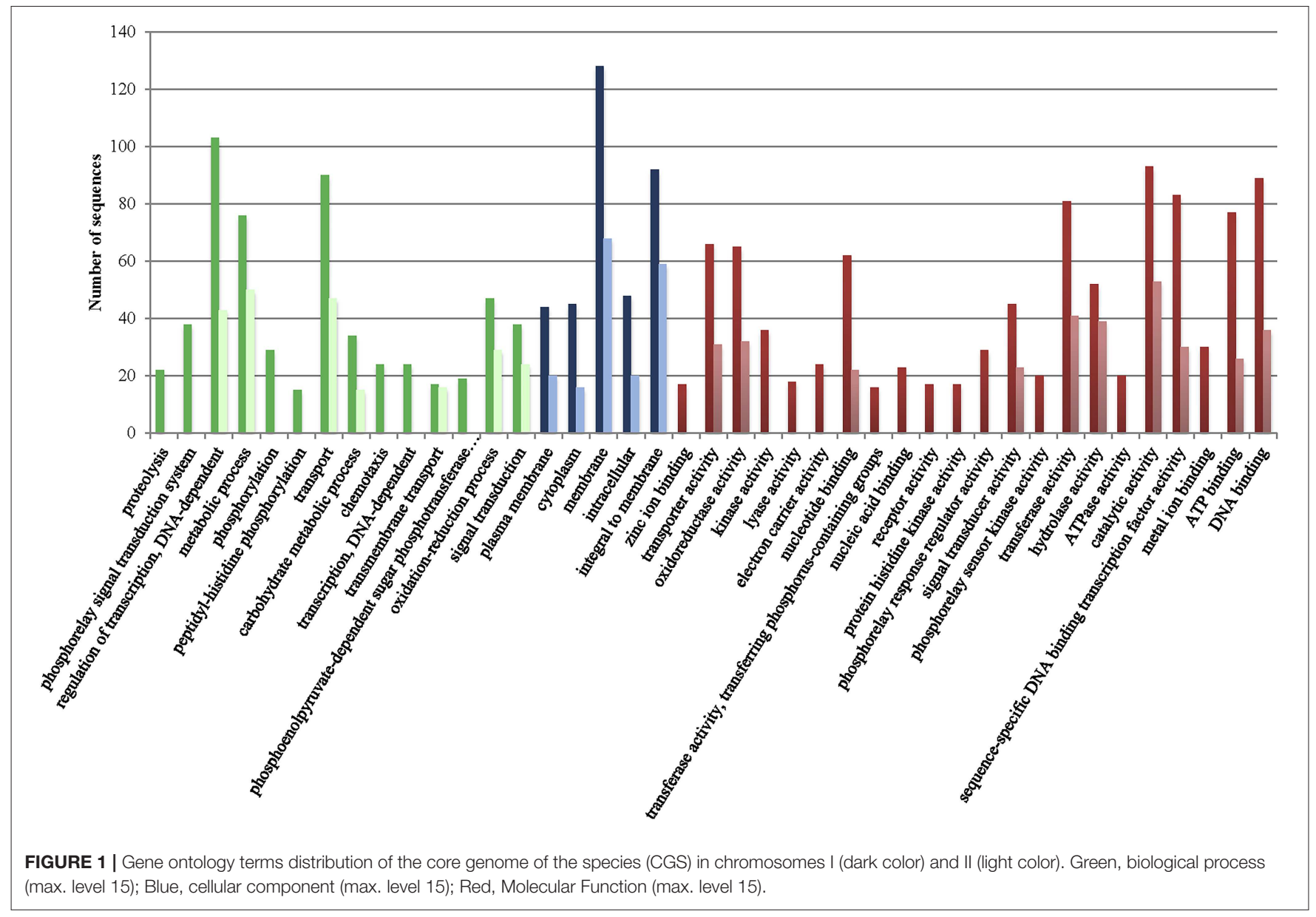

TABLE 4 | Virulence plasmid types and main characteristics.

\begin{tabular}{|c|c|c|c|c|c|c|c|}
\hline pVvbt2 type & Strains* & $\begin{array}{l}\% \text { identity whole } \\
\text { sequence }\end{array}$ & $\%$ identity core & Max size & Min size & Core size & GC \\
\hline I & 95-87, 95-86, A14 & 98.6 & 100 & 65,910 & 65,375 & 46,446 & 43.4 \\
\hline III & $94-8-112,96-0426-1-4 C$ & 81.5 & 99.7 & 64,359 & 63,933 & 46,446 & 43.8 \\
\hline IV & $\begin{array}{l}\text { CECT898, CECT4865, PD-2-51, Rae3, } \\
\text { CECT4604, CECT4866, CIP8190 }\end{array}$ & 88.1 & 100.0 & 68,448 & 66,946 & 46,446 & 43.5 \\
\hline VI & $95-8-161$ & 100 & 100 & 63,933 & 63,933 & 46,446 & 43.3 \\
\hline
\end{tabular}

*Serovar Estrains: R02, CECT4999, CECT5763, 94-8-112, CECT898, CECT4865, PD-2-51, Rae3, CECT4604, CECT4866, CIP8190. Serovar A strains: CECT7030, CECT5769, A14. Serovar I, 95-87, 95-86, 95-8-161. Non typable, 96-0426-1-4C.

another, which strongly suggests that both chromosomes have suffered inter and/or intra chromosomal rearrangement since the emergence of the $V$. vulnificus ancestor. In fact, several strains changed their relationships in the tree for each chromosome (Figure 2). Among them, we highlight the wellknown human clinical isolates C7184, YJ016, and CMCP6 (Figure 2). Despite the global incongruence between the two chromosomal phylogenies, all the phylogenetic trees divided the species into five well-defined, highly supported lineages, two of them (L4 and 5) including only a few strains. No strain changed lineage between the two trees (Figure 2).

The pairwise identity for the strains of all the lineages was $82.5 \%$ (Table 6). Lineage 1 (L1) (pairwise identity 93.1\%) includes clinical ( $50 \%$ of isolates) and environmental ( $50 \%$ of isolates) Bt1 isolates from the USA, South Korea, Taiwan, Israel, and Spain. The clinical L1 isolates were recovered from human infections in the USA, South Korea, and Taiwan, with $80 \%$ of these derived from blood (related to primary septicemia, when the etiology 

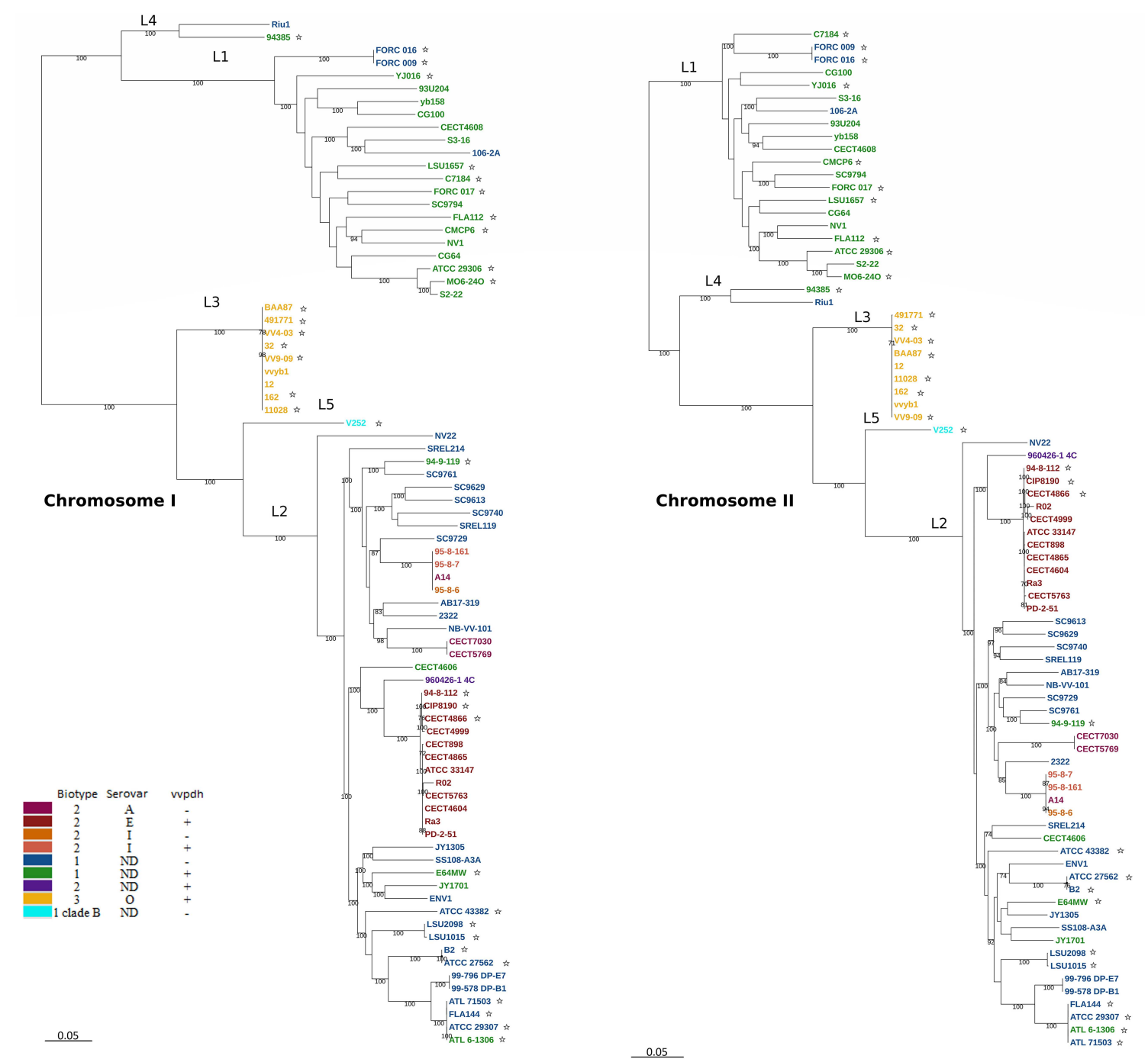

FIGURE 2 | V. vulnificus phylogeny reconstructed from single nucleotide polymorphisms (SNPs) of the coding regions in the CGS. V. vulnificus phylogeny based on single nucleotide polymorphisms (SNPs) of the coding regions in the core genome of the species (CGS). Maximum-likelihood tree derived using the generalized time-reversible model $(G T R+G+I)$ model of evolution. Bootstrap support values higher than $70 \%$ are indicated in the corresponding nodes. *Human clinical isolate.

is known), $10 \%$ from stools (related to gastroenteritis) and $10 \%$ from wound samples. The environmental L1 isolates were recovered from oysters (40\%), water (30\%), and fish (30\%) in Spain, Israel, Taiwan, South Korea, and the USA. Interestingly, the strain yv158, although environmental, belongs to a previously described highly clonal group (clade A), which includes both environmental and clinical isolates (wound samples), all of $v c g$ C-type (clinical type according to the polymorphism in virulence correlated gene; Rosche et al., 2005), supporting the potential virulence of this specific strain (Broza et al., 2012).

L2 (pairwise identity $87.4 \%$ ) includes Bt1 and Bt2 strains from environmental sources $(39.6 \%$ of the isolates; $21 \%$ from water, $21 \%$ from fish, $53.5 \%$ from seafood and $4.5 \%$ from sediment), diseased humans [ $29.2 \%$ of the isolates; $57 \%$ from human blood (none of them with known etiology) and 43\% from wounds] and diseased fish (31.2\%) origins. All of the zoonotic strains (Bt2SerE), regardless of their source (environment, diseased animal or diseased human), country (France, Australia, Denmark and Spain) or year of isolation (from 1980 to 2004), cluster in a highly homogeneous group (pairwise identity 97.7\%). The non-typeable Bt2 strain (960426-1 4C) formed a subgroup together with the SerE clonal-complex while SerI and A strains formed another subgroup both within L2 (Figure 2).

L3 includes all the Bt3 strains, which form a highly homogeneous group (pairwise identity 97.8\%). These Bt3 strains were isolated in Israel from outbreaks of human vibriosis associated with the handling of farmed-tilapia (year of isolation, 1996-2003) and from aquaculture fishponds of tilapia 
TABLE 5 | Summary of Shimodaira-Hasegawa (SH) and Expected Likelihood Weight (ELW) tests.

\begin{tabular}{|c|c|c|c|c|c|c|c|c|c|c|}
\hline \multirow{2}{*}{$\begin{array}{l}\text { Alignment } \\
\text { Chrl }\end{array}$} & \multirow{2}{*}{$\begin{array}{l}\text { Topology } \\
\text { Chrl }\end{array}$} & \multirow{2}{*}{$\begin{array}{l}\text { log-L } \\
-2157881.655\end{array}$} & \multicolumn{2}{|c|}{ p-1sKH } & \multicolumn{2}{|c|}{ p-SH } & \multicolumn{2}{|c|}{ c-ELW } & \multicolumn{2}{|c|}{ p-AU } \\
\hline & & & 1.000 & + & 1.000 & + & 1.000 & + & 1.000 & + \\
\hline & Chrll & -2179900.127 & 0.000 & - & 0.000 & - & 0.000 & - & 0.000 & - \\
\hline \multirow[t]{2}{*}{ Chrll } & Chrl & -761721.329 & 0.000 & - & 0.000 & - & 0.000 & - & 0.000 & - \\
\hline & Chrll & -757275.779 & 1.000 & + & 1.000 & + & 1.000 & + & 1.000 & + \\
\hline \multirow[t]{2}{*}{ Virulence Plasmid } & Chrl & -73205.493 & 0 & - & 0 & - & 0 & - & 0.0019 & - \\
\hline & Chrll & -73221.068 & 0 & - & 0 & - & 0 & - & 0.0009 & - \\
\hline Virulence Plasmid & Virulence Plasmid & -72555.771 & 1.000 & + & 1.000 & + & 1.000 & + & 0.9977 & + \\
\hline \multirow[t]{3}{*}{ Chrl } & Chrl & -340807.458 & 0.942 & + & 1.000 & + & 0.955 & + & 0.972 & + \\
\hline & Chrll & -340840.343 & 0.058 & + & 0.496 & + & 0.0454 & - & 0.028 & - \\
\hline & Virulence Plasmid & -405217.661 & 0 & - & 0 & - & 0 & - & 0.005 & - \\
\hline \multirow[t]{3}{*}{ Chrll } & Chrl & -149737.006 & 0.043 & - & 0.472 & + & 0.014 & - & 0.078 & - \\
\hline & Chrll & -149703.356 & 0.957 & + & 1.000 & + & 0.986 & + & 0.998 & + \\
\hline & Virulence Plasmid & -178030.177 & 0 & - & 0 & - & 0 & - & 0.0066 & - \\
\hline
\end{tabular}

The columns show the results and p-values of the following tests: 1sKH-one sided KH test based on pairwise SH tests (Kishino and Hasegawa, $1989 ;$ Shimodaira and Hasegawa, 1999;

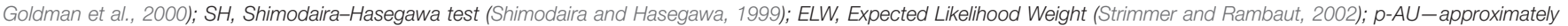
unbiased test (Shimodaira, 2002). $p<0.05 .+$, positive congruence; -, negative congruence.

TABLE 6 | Global identity of groups and lineages.

\begin{tabular}{lc}
\hline Name & \% Pairwise Identity \\
\hline Whole & 82.5 \\
Biotype 3 & 97.8 \\
Biotype 2 & 94.8 \\
Lineage 1 & 93.1 \\
Lineage 2 & 87.4 \\
Lineage 3 & 97.8 \\
Lineage 4 & 98.7 \\
Lineage 5 & 100 \\
Biotype 2 SerA & 91.0 \\
Biotype 2 SerE & 97.7 \\
Biotype 2 Serl & 100.0 \\
\hline
\end{tabular}

(2002-2004). In consequence, all of them are associated to the aquaculture industry.

Interestingly, all human clinical cases of known etiology caused by L2 and L3 isolates in Europe and Israel were related to farmed-fish handling regardless the $\mathrm{Bt}$ of the isolate.

L4 (similarity $98.7 \%$ ) is formed by two Spanish isolates from the Ebro-Delta (a nature park by the Mediterranean Sea) area, one from seawater and the other from a human clinical (leg wound) case, both of Bt1. Finally, L5 is formed by a unique isolate of Bt1 from Israel and clinical origin that is considered as representative of a highly virulent clone designated as Clade B (Raz et al., 2014; Efimov et al., 2015).

The phylogenetic tree for $\mathrm{pVvBt} 2$ based on the CGP shows relationships among isolates that did not match the previous phylogenetic relationships (Figures 2, 3). Further, the congruence analyses revealed that phylogenetic trees from the CGP and CGS were not congruent to each other, suggesting a different evolutionary history for the plasmid and the chromosomes (Table 5).

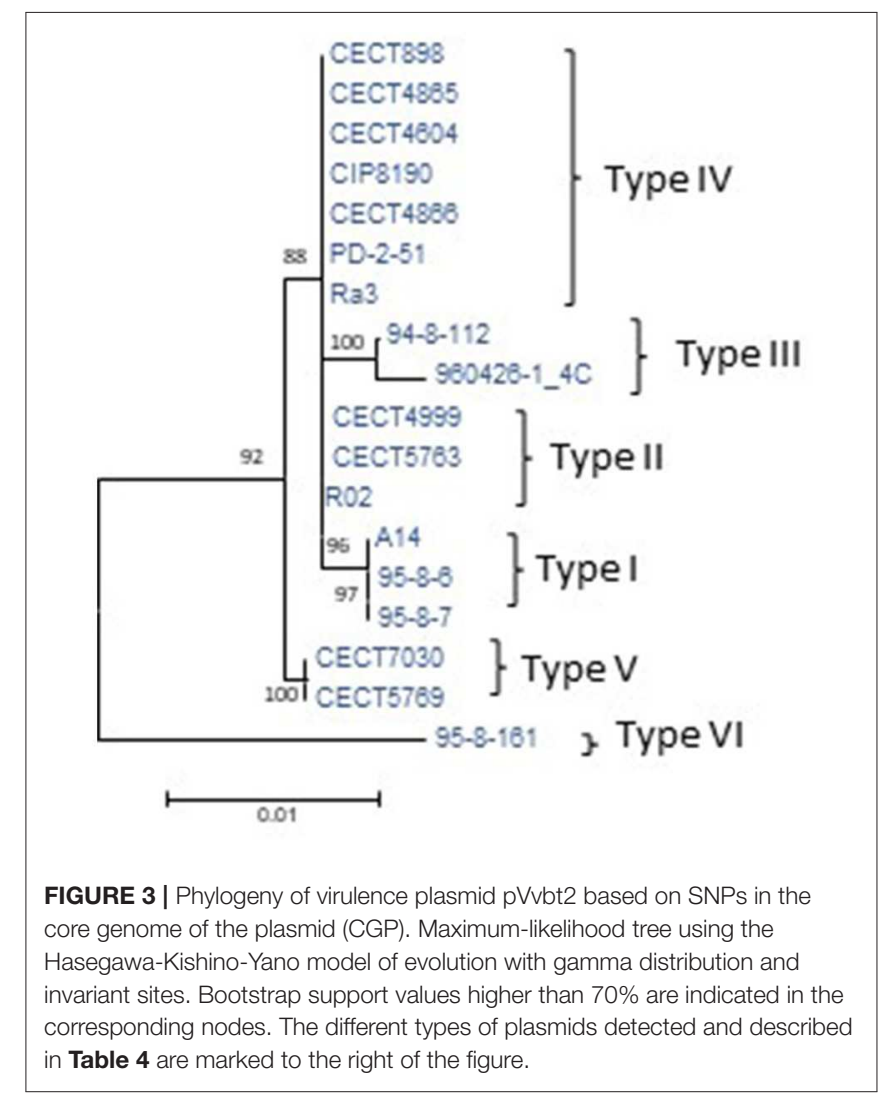

\section{Virulence-Related Genes and VCGS}

We searched for human virulence-related genes described in the literature for $V$. vulnificus, and we found that $75 \%$ of them were present in the CGS. This finding allowed us to define the human virulence-related core genome (VCGS) (Table S6). The VCGS includes genes for the flagellum, capsule, LPS- 
and cell-wall biosynthesis, motility, hemolysin, and proteases (such as VvhA and a collagenase), resistance to human serum (trkA; Chen et al., 2004), heme uptake, biosynthesis and uptake of vulnibactin, several genes for transcriptional regulators such as Fur, and finally genes for MARTX (Multifunctional Autoprocessive Repeat in Toxin) transport and modification systems, genes that are duplicated in pVvbt2 (Chakrabarti et al., 1999; Horstman et al., 2004; Yu-Chung et al., 2004; Gulig et al., 2005; Bogard and Oliver, 2007; Alice et al., 2008; Lee et al., 2008; Oh et al., 2008; Brown and Gulig, 2009; Liu et al., 2009; Chen and Chung, 2011; Kim et al., 2011; Oliver, 2015; Satchell, 2015). Remarkably, genes for several collagenases and chitinases were found in both chromosomes while the MARTX operon and $v v h A$ were located on chromosome II.

\section{DISCUSSION}

The core genome has been demonstrated to be an optimum data set for determining the phylogeny of a bacterial species since it primarily includes the essential genes of a species and excludes the non-essential ones, such as those present in MGE (mobile genetic element: genomic islands, prophages and plasmids) (Juhas et al., 2009; Segerman, 2012; Wolf et al., 2013). In the current study, we have used this approach to analyze the phylogeny of $V$. vulnificus and compare the phylogenetic groups with the current Bts of the species.

Our phylogenomic analysis suggests that $V$. vulnificus has diverged in five well-defined and separate lineages that do not correspond with the current Bts. L1 is formed by the most dangerous strains from a public health perspective. All of them correspond to Bt 1 and were mostly isolated from human blood in North America and Asia, presumably from primary septicemia cases after ingestion of raw seafood. L2 and L3 comprise strains of the three Bts, mostly isolated from fish-farming related environments, including humans infected through handling of farm-fish in Europe and Israel (Bisharat et al., 1999; Haenen et al., 2013).

L2 includes Bt1 and Bt2 strains. Sanjuán et al. (2011) proposed that Bt2 is a polyphyletic group subdivided in Serrelated subgroups, one of which is a clonal complex (Bt2-SerE). Our phylogenomic study confirms that Bt2 is polyphyletic and that the SerE-subgroup is highly homogeneous (identity value of 97.7\%). Bt2 was defined in 1982 based on the differential properties of the first fish-pathogenic strains, all of which belonged to SerE and were isolated from eel-farms in Japan (Muroga et al., 1976a,b; Tison et al., 1982). Later, Bt2-SerE isolates were recovered from human infections registered in the USA and Europe, some of them related to zoonotic cases and others of unknown etiology, as well as from different epizootic events of high mortality affecting different farmed-eels in Europe. Bt2SerA and SerI emerged simultaneously in Spanish and Danish farms after the industry initiated the change from brackish- to fresh-waters in order to control the severity of vibriosis outbreaks due to Bt2-SerE as well as the probability of human infections (Fouz and Amaro, 2003). These new serovars are adapted to infect through and to survive in fresh-waters (Fouz et al., 2006).
All of the analyzed Bt2 strains contained the virulence plasmid $p V v b t 2$, SerE strains present three variants of the plasmid, the two variants previously described (Lee et al., 2008) and a new one (Table 4). SerA and I strains showed three new variants (Table 4). It was previously hypothesized that Bt2 emerged in fish farms after acquisition of pVvbt2 by different clones of Bt1 strains (Sanjuán et al., 2011). To test this hypothesis, we compared the chromosomal phylogenetic trees reconstructed for Bt2 strains from the CGS with those from the CGP and found that they were not congruent. This result strongly supports the hypothesis of Sanjuán et al. (2011) and suggests that pVvbt2 has been acquired independently by different clones within L2. One of these plasmid-carrier clones successfully amplified in eelfarms and spread to other places and countries, probably in carrier fishes, giving rise to the worldwide expanded, current clonal complex. This clonal complex is supposed to be zoonotic because there are clinical Bt2-SerE isolates related to diseased fish handling and because all the fish and environmental isolates examined to date are virulent for both fish and mice (Sanjuán and Amaro, 2004).

L3 includes all Bt3 strains regardless of their origin (human infections related to fish-farms or environmental), which constitute a clonal group. Bt3 emerged in Israel in 1990 in farms of tilapia and is the only one that has produced outbreaks of human infections, all of them through severe wound infections or secondary septicemia cases (Bisharat et al., 1999). By using different genomic approaches, Raz et al. (2014) and Koton et al. (2014) have hypothesized that Bt3 emerged in the nutrientenriched environment represented by the aquaculture industry from a Bt1 ancestor that acquired a rather small number of genes from different donors, leading to a change in biotype. The proposed ancestor was v252, a representative strain from a highly virulent clade designated as clade B that shares high similarity and appeared close to Bt3 (Raz et al., 2014; Efimov et al., 2015). Our analysis does not support this hypothesis. Instead, clade B shares the closest common ancestor with L2 and not with L3 in spite of having been isolated from the same "melting pot" where biotype 3 was evolved, i.e., aquaculture fish farms in Israel.

Comparisons of the core genome between clinical and environmental strains of the closely related species $V$. cholerae reveal that this species is divided into two linages, with most of the epidemic strains appearing closely related, regardless of their geographical origins (Eppinger et al., 2014). The only clonal $V$. vulnificus group with a worldwide distribution is that formed by Bt2-SerE strains, a group that combines the ability to infect fish with that of infecting humans and of surviving in the environment without nutrients for years in a viable but non-culturable state (Marco-Noales et al., 1999). Moreover, in $V$. cholerae the ability to cause cholera epidemics lies on mobile genetic elements, such as phages and pathogenicity islands, that carry the genes encoding the cholera toxin, TCP pilus, etc. (Ramamurthy and Bhattacharya, 2011; Das et al., 2016). In contrast, our phylogenomic analysis, as well as those based on MLSA (Cohen et al., 2007; Sanjuán et al., 2011) and microarray hybridization (Raz et al., 2014), show that environmental and clinical strains of $V$. vulnificus are distributed throughout the phylogenetic lineages, regardless the $\mathrm{Bt}$, country of origin, or year 
of isolation. This result is compatible with the hypothesis that essentially all $V$. vulnificus isolates, unlike $V$. cholerae, have the ability to infect humans. To confirm this, we investigated which virulence-related genes were present in the CGS and found that most of them belong to the core genome.

Summarizing, all of the phylogenetic reconstructions from the core genome of the species, the fish-virulence plasmid and the human-virulence genes strongly suggest that $V$. vulnificus emerged from an ancestor potentially virulent for humans that diverged in five lineages that do not correspond with the current Bts. Our results also highlight the importance of the aquaculture industry in the recent evolution and epidemic spread of the species and, finally, support the intra-specific classification in lineages instead of in Bts as well as the inclusion of a pathovar grouping all fish pathogenic isolates for which we propose the name "piscis."

\section{AUTHOR CONTRIBUTIONS}

CA, FG-C, EF, and FR designed the work, FR, ES, and FG-C performed the phylogenomic analysis, YD-P, BF, CG, CB-A, PG, and $\mathrm{SM}$ discussed the preliminary results. $\mathrm{CA}$ and $\mathrm{FR}$ wrote the paper. All the authors contributed to the discussion and improvement of the MS.

\section{FUNDING}

This work has been financed by grants AICO/2018/123, AGL2017-87723-P (both co-funded with FEDER funds), BFU2014-58656-R, Programa Consolider-Ingenio 2010 CSD2009-00006 from MICINN (Spain), and PROMETEO/2016/122 from Generalitat Valenciana.

\section{REFERENCES}

Akaike, H. (1974). A new look at the statistical model identification. IEEE Trans. Automat. Contr. 19, 716-723. doi: 10.1109/TAC.1974.1100705

Alice, A. F., Naka, H., and Crosa, J. H. (2008). Global gene expression as a function of the iron status of the bacterial cell: influence of differentially expressed genes in the virulence of the human pathogen Vibrio vulnificus. Infect. Immun. 76, 4019-4037. doi: 10.1128/IAI.00208-08

Amaro, C., Sanjuán, E., Fouz, B., Pajuelo, D., Lee, C.-T., Hor, L.-L., et al. (2015). The fish pathogen Vibrio vulnificus Biotype 2: epidemiology, phylogeny, and virulence factors involved in warm-water vibriosis. Microbiol. Spectr. 3:VE0005-2014. doi: 10.1128/microbiolspec.VE-0005-2014

Bairoch, A. (2000). The ENZYME database in 2000. Nucleic Acids Res. 28, 304-305. doi: 10.1093/nar/28.1.304

Baker-Austin, C., Trinanes, J. A., Taylor, N. G. H., Hartnell, R., Siitonen, A., and Martinez-Urtaza, J. (2013). Emerging Vibrio risk at high latitudes in response to ocean warming. Nat. Clim. Chang. 3, 73-77. doi: 10.1038/nclimate1628

Baker-Austin, C., Trinanes, J., Gonzalez-Escalona, N., and Martinez-Urtaza, J. (2017). Non-Cholera Vibrios: the microbial barometer of climate change. Trends Microbiol. 25, 76-84. doi: 10.1016/j.tim.2016.09.008

Bankevich, A., Nurk, S., Antipov, D., Gurevich, A. A., Dvorkin, M., Kulikov, A. S., et al. (2012). SPAdes: a new genome assembly algorithm and its applications to single-cell sequencing. J. Comput. Biol. 19, 455-477. doi: $10.1089 / \mathrm{cmb} .2012 .0021$

Biosca, E. G., Amaro, C., Larsen, J. L., and Pedersen, K. (1997). Phenotypic and genotypic characterization of Vibrio vulnificus - proposal for the substitution

\section{ACKNOWLEDGMENTS}

The authors also thank the SCSIE of the University of Valencia for technical support in determining the sequences.

\section{SUPPLEMENTARY MATERIAL}

The Supplementary Material for this article can be found online at: https://www.frontiersin.org/articles/10.3389/fmicb. 2017.02613/full\#supplementary-material

Figure S1 | Vibrio phylogeny based on the concatenated whole core genome of seven Vibrio species with closed genomes together with selected $V$. vulnificus strains for chromosome I, chromosome II and chromosome I+II.

Maximum-likelihood tree derived from the aligned regions by using the $\mathrm{GTR}+\mathrm{G}+\mathrm{I}$ model of evolution. Bootstrap support values higher than $70 \%$ are indicated in the corresponding nodes. Color code: green, Bt1 vvpdh+; blue; Bt1 vvpdh-; yellow; Bt3 vvpdh+; red; Bt2 vvpdh+; magenta; Br2 vvpdh-.

Figure S2 | V. vulnificus phylogeny reconstructed from single nucleotide polymorphisms (SNPS) of the coding regions in the CGS for both chromosmes (Chrl+Chrll). V. vulnificus phylogeny based on single nucleotide polymorphisms (SNPs) of the coding regions in the core genome of the species (CGS). Maximum-likelihood tree derived using the generalized time-reversible model $(\mathrm{GTR}+\mathrm{G}+\mathrm{l})$ model of evolution. Bootstrap support values higher than $70 \%$ are indicated in the corresponding nodes. *Human clinical isolate.

Table S1 | Characteristics of the Vibrio genomes used to define the core genome of the genus (CGG) according to the NCBI Databases.

Table S2 | V. vulnificus core genome.

Table S3 | Metabolic pathways associated to V. vulnificus core genome.

Table S4 | V. vulnificus common genes that were not considered to be part of the Core Genome because its identity and length with respect to the reference sequence were lower than 70 and $80 \%$, respectively.

Table S5 | Core genes in the V. vulnificus plasmid pVvbt2 (CGP).

Table S6 | V. vulnificus virulence genes in the core genome.

of the subspecific taxon biotype for serovar. Appl. Environ. Microbiol. 63, 1460-1466.

Bisharat, N., Agmon, V., Finkelstein, R., Raz, R., Ben-Dror, G., Lerner, L., et al. (1999). Clinical, epidemiological, and microbiological features of Vibrio vulnificus biogroup 3 causing outbreaks of wound infection and bacteraemia in Israel. Lancet 354, 1421-1424. doi: 10.1016/S0140-6736(99) 02471-X

Bogard, R. W., and Oliver, J. D. (2007). Role of iron in human serum resistance of the clinical and environmental Vibrio vulnificus genotypes. Appl. Environ. Microbiol. 73, 7501-7505. doi: 10.1128/AEM.01551-07

Brown, R. N., and Gulig, P. A. (2009). Roles of RseB, sigmaE, and DegP in virulence and phase variation of colony morphotype of Vibrio vulnificus. Infect. Immun. 77, 3768-3781. doi: 10.1128/IAI.00205-09

Broza, Y. Y., Raz, N., Lerner, L., Danin-Poleg, Y., and Kashi, Y. (2012). Genetic diversity of the human pathogen Vibrio vulnificus: a new phylogroup. Int. J. Food Microbiol. 153, 436-443. doi: 10.1016/j.ijfoodmicro.2011.12.011

Chakrabarti, S., Sengupta, N., and Chowdhury, R. (1999). Role of DnaK in in vitro and in vivo expression of virulence factors of Vibrio cholerae. Infect. Immun. 67, 1025-1033.

Chen, C.-Y., Wu, K.-M., Chang, Y.-C., Chang, C.-H., Tsai, H.-C., Liao, T.-L., et al. (2003). Comparative genome analysis of Vibrio vulnificus, a marine pathogen. Genome Res. 13, 2577-2587. doi: 10.1101/gr.1295503

Chen, Y.-C., Chuang, Y.-C., Chang, C.-C., Jeang, C.-L., and Chang, M.-C. (2004). A K+ uptake protein, TrkA, is required for serum, protamine, and polymyxin B Resistance in Vibrio vulnificus. Infect. Immun. 72, 629-636. doi: 10.1128/IAI.72.2.629-636.2004 
Chen, Y. C., and Chung, Y. T. (2011). A conserved GTPase YchF of Vibrio vulnificus is involved in macrophage cytotoxicity, iron acquisition, and mouse virulence. Int. J. Med. Microbiol. 301, 469-474. doi: 10.1016/j.ijmm.2011.02.002

Cohen, A. L. V., Oliver, J. D., DePaola, A., Feil, E. J., Boyd, E. F., Fidelma Boyd, E., et al. (2007). Emergence of a virulent clade of Vibrio vulnificus and correlation with the presence of a 33-kilobase genomic island. Appl. Environ. Microbiol. 73, 5553-5565. doi: 10.1128/AEM.00635-07

Danin-Poleg, Y., Raz, N., Roig, F. J., Amaro, C., Kashi, Y. (2015). Draft genome sequence of environmental bacterium Vibrio vulnificus CladeA-yb158. Genome Announc. 3:e00754-15. doi: 10.1128/genomeA.00754-15

Darling, A. C. E., Mau, B., Blattner, F. R., and Perna, N. T. (2004). Mauve: multiple alignment of conserved genomic sequence with rearrangements. Genome Res. 14, 1394-1403. doi: 10.1101/gr.2289704

Das, B., Pazhani, G. P., Sarkar, A., Mukhopadhyay, A. K., Nair, G. B., and Ramamurthy, T. (2016). Molecular evolution and functional divergence of Vibrio cholerae. Curr. Opin. Infect. Dis. 29, 520-527. doi: 10.1097/QCO.0000000000000306

Efimov, V., Danin-Poleg, Y., Green, S., Elgavish, S., and Kashi, Y. (2015). Draft genome sequence of the pathogenic bacterium Vibrio vulnificus V252 biotype 1, isolated in Israel. Genome Announc. 3:e01182-15. doi: 10.1128/genomeA.01182-15

Eppinger, M., Pearson, T., Koenig, S. S. K., Pearson, O., Hicks, N., Agrawal, S., et al. (2014). Genomic epidemiology of the Haitian cholera outbreak: A single introduction followed by rapid, extensive, and continued spread characterized the onset of the epidemic. MBio 5:e1721-14. doi: 10.1128/mBio.01721-14

Farmer, J. J. III. (1979). Vibrio ("Beneckea") vulnificus, the bacterium associated with sepsis, septicaemia, and the sea. Lancet 2, 903.

Felsenstein, J. (1985). Confidence limits on phylogenies: an approach using the bootstrap. Evolution 39, 783-791.

Fouz, B., and Amaro, C. (2003). Isolation of a new serovar of Vibrio vulnificus pathogenic for eels cultured in freshwater farms. Aquaculture 217, 677-682. doi: 10.1016/S0044-8486(02)00200-4

Fouz, B., Larsen, J. L., and Amaro, C. (2006). Vibrio vulnificus serovar A: an emerging pathogen in European anguilliculture. J. Fish Dis. 29, 285-291. doi: 10.1111/j.1365-2761.2006.00719.x

Futami, R., Muñoz-Pomer, L., Viu, J. M., Domínguez-Escribá, L., Covelli, L., Bernet, G. P., et al. (2011). GPRO: the professional tool for management, functional analysis and annotation of omic sequences and databases. Biotech. Bioinform. 2011-SOFT3.

Gene Ontology Consortium (2008). The Gene Ontology project in 2008. Nucleic Acids Res. 36, D440-D444. doi: 10.1093/nar/gkm883

Goldman, N., Anderson, J. P., Rodrigo, A. G. (2000). Likelihoodbased tests of topologies in phylogenetics. System. Biol. 49, 652-670. doi: $10.1080 / 106351500750049752$

Guindon, S., Delsuc, F., Dufayard, J. F., and Gascuel, O. (2009). Estimating maximum likelihood phylogenies with PhyML. Methods Mol. Biol. 537, 113-137. doi: 10.1007/978-1-59745-251-9_6

Gulig, P. A., Bourdage, K. L., and Starks, A. M. (2005). Molecular Pathogenesis of Vibrio vulnificus. J. Microbiol. 43, 118-131.

Haenen, O. L. M., Evans, J. J., and Berthe, F. (2013). Bacterial infections from aquatic species: potential for and prevention of contact zoonoses. Rev. Off. Int. Epizoot. 32, 497-507. doi: 10.20506/rst.32.2.2245

Harris, S. R., Feil, E. J., Holden, M. T. G., Quail, M. A., Nickerson, E. K., Chantratita, N., et al. (2010). Evolution of MRSA during hospital transmission and intercontinental spread. Science 327, 469-474. doi: $10.1126 /$ science. 1182395

Hasegawa, M., Kishino, H., and Yano, T. (1985). Dating of the human-ape splitting by a molecular clock of mitochondrial DNA. J. Mol. Evol. 22, 160-174. doi: 10.1007/BF02101694

Hlady, W. G., and Klontz, K. C. (1996). The epidemiology of Vibrio infections in Florida, 1981-1993. J. Infect. Dis. 173, 1176-1183. doi: 10.1093/infdis/173.5.1176

Horstman, A. L., Bauman, S. J., and Kuehn, M. J. (2004). Lipopolysaccharide 3Deoxy-D-manno-octulosonic Acid (Kdo) core determines bacterial association of secreted toxins. J. Biol. Chem. 279, 8070-8075. doi: 10.1074/jbc.M3086 33200

Jones, M. K., and Oliver, J. D. (2009). Vibrio vulnificus: disease and pathogenesis. Infect. Immun. 77, 1723-1733. doi: 10.1128/IAI.01046-08
Juhas, M., van der Meer, J. R., Gaillard, M., Harding, R. M., Hood, D. W., and Crook, D. W. (2009). Genomic islands: tools of bacterial horizontal gene transfer and evolution. FEMS Microbiol. Rev. 33, 376-393. doi: 10.1111/j.1574-6976.2008.00136.x

Katoh, K., and Standley, D. M. (2013). MAFFT multiple sequence alignment software version 7: improvements in performance and usability. Mol. Biol. Evol. 30, 772-780. doi: 10.1093/molbev/mst010

Kim, I. H., Kim, B. S., Lee, K. S., Kim, I. J., Son, J. S., and Kim, K. S. (2011). Identification of virulence factors in Vibrio vulnificus by comparative transcriptomic analyses between clinical and environmental isolates using cDNA microarray. J. Microbiol. Biotechnol. 21, 1228-1235. doi: 10.4014/jmb.1111.11016

Kirkup, B. C., Chang, L., Chang, S., Gevers, D., and Polz, M. F. (2010). Vibrio chromosomes share common history. BMC Microbiol. 10:137. doi: 10.1186/1471-2180-10-137

Kishino, H., and Hasegawa, M. (1989). Evaluation of the maximum likelihood estimate of the evolutionary tree topologies from DNA sequence data, and the branching order in Hominoidea. J. Mol. Evolut. 29, 170-179. doi: 10.1007/BF02100115

Kotera, M., Hirakawa, M., Tokimatsu, T., Goto, S., and Kanehisa, M. (2012). The KEGG databases and tools facilitating omics analysis: latest developments involving human diseases and pharmaceuticals. Methods Mol. Biol. 802, 19-39. doi: 10.1007/978-1-61779-400-1_2

Koton, Y., Gordon, M., Chalifa-Caspi, V., and Bisharat, N. (2014). Comparative genomic analysis of clinical and environmental Vibrio vulnificus isolates revealed biotype 3 evolutionary relationships. Front. Microbiol. 5:803. doi: $10.3389 /$ fmicb.2014.00803

Lee, C.-T., Amaro, C., Wu, K.-M., Valiente, E., Chang, Y.-F., Tsai, S.-F., et al. (2008). A common virulence plasmid in biotype 2 Vibrio vulnificus and its dissemination aided by a conjugal plasmid. J. Bacteriol. 190, 1638-1648. doi: 10.1128/JB.01484-07

Lee, C.-T., Pajuelo, D., Llorens, A., Chen, Y.-H., Leiro, J. M., Padrós, F., et al. (2013a). MARTX of Vibrio vulnificus biotype 2 is a virulence and survival factor. Environ. Microbiol. 15, 419-432. doi: 10.1111/j.1462-2920.2012. 02854.x

Lee, K. J., Lee, N. Y., Han, Y. S., Kim, J., Lee, K. H., and Park, S. J. (2010). Functional characterization of the IlpA protein of Vibrio vulnificus as an adhesin and its role in bacterial pathogenesis. Infect. Immun. 78, 2408-2417. doi: 10.1128/IAI.01194-09

Lee, S. H., Chung, B. H., and Lee, W. C. (2013b). Retrospective analysis of epidemiological aspects of Vibrio vulnificus infections in Korea in 2001-2010. Jpn. J. Infect. Dis. 66, 331-333. doi: 10.7883/yoken.66.331

Liu, M., Naka, H., and Crosa, J. H. (2009). HlyU acts as an H-NS antirepressor in the regulation of the RTX toxin gene essential for the virulence of the human pathogen Vibrio vulnificus CMCP6. Mol. Microbiol. 72, 491-505. doi: 10.1111/j.1365-2958.2009.06664.x

Marco-Noales, E., Biosca, E. G., and Amaro, C. (1999). Effects of salinity and temperature on long-term survival of the eel pathogen Vibrio vulnificus biotype 2 (serovar E). Appl. Environ. Microbiol. 65, 1117-1126.

Marco-Noales, E., Milán, M., Fouz, B., Sanjuán, E., Amaro, C., Milan, M., et al. (2001). Transmission to eels, portals of entry, and putative reservoirs of Vibrio vulnificus Serovar E (Biotype 2). Appl. Environ. Microbiol. 67, 4717-4725. doi: 10.1128/AEM.67.10.4717-4725.2001

Muroga, K., Jo, Y., and Nishibuchi, M. (1976a). Pathogenic Vibrio isolated from cultured eels. I. Characteristics and taxonomic status. Fish Pathol. 11, 141-145. doi: $10.3147 /$ jsfp. 11.141

Muroga, K., Nishibuchi, M., and Jo, Y. (1976b). Pathogenic Vibrio isollated from cultured eels. II. Physiological characteristics and pathogenicity. Fish Pathol. 11, 147-151. doi: $10.3147 /$ jsfp.11.147

Newton, A., Kendall, M., Vugia, D. J., Henao, O. L., and Mahon, B. E. (2012). Increasing rates of vibriosis in the United States, 1996-2010: review of surveillance data from 2 systems. Clin. Infect. Dis. 54, S391-S395. doi: $10.1093 / \mathrm{cid} / \mathrm{cis} 243$

Oh, M. H., Jeong, H. G., and Choi, S. H. (2008). Proteomic identification and characterization of Vibrio vulnificus proteins induced upon exposure to INT407 intestinal epithelial cells. J. Microbiol. Biotechnol. 18, 968-974.

Oliver, J. D. (2015). The biology of Vibrio vulnificus. Microbiol. Spectr. 3:VE-00012014. doi: 10.1128/microbiolspec.VE-0001-2014 
Pajuelo, D., Lee, C.-T., Roig, F. J., Hor, L.-I., and Amaro, C. (2015). Novel hostspecific iron acquisition system in the zoonotic pathogen Vibrio vulnificus. Environ. Microbiol. 17, 2076-2089. doi: 10.1111/1462-2920.12782

Posada, D. (2008). jModelTest: phylogenetic model averaging. Mol. Biol. Evol. 25, 1253-1256. doi: 10.1093/molbev/msn083

Ramamurthy, T., and Bhattacharya, S. K. (2011). Epidemiological and Molecular Aspects on Cholera. New York, NY: Springer-Verlag.

Raz, N., Danin-Poleg, Y., Hayman, R. B., Bar-On, Y., Linetsky, A., Shmoish, M., et al. (2014). Genome-wide SNP-genotyping array to study the evolution of the human pathogen Vibrio vulnificus biotype 3. PLoS ONE 9:e114576. doi: 10.1371/journal.pone. 0114576

Rippey, S. R. (1994). Infectious diseases associated with molluscan shellfish consumption. Clin. Microbiol. Rev. 7, 419-425. doi: 10.1128/CMR.7.4.419

Roig, F. J., and Amaro, C. (2009). Plasmid diversity in Vibrio vulnificus biotypes. Microbiology 155, 489-497. doi: 10.1099/mic.0.023424-0

Roig, F. J., González-Candelas, F., Amaro, C., Gonzalez-Candelas, F., and Amaro, C. (2011). Domain organization and evolution of multifunctional autoprocessing repeats-in-toxin (MARTX) toxin in Vibrio vulnificus. Appl. Environ. Microbiol. 77, 657-668. doi: 10.1128/AEM.01806-10

Roig, F. J., Sanjuán, E., Llorens, A., and Amaro, C. (2010). pilF polymorphism-based PCR to distinguish Vibrio vulnificus strains potentially dangerous to public health. Appl. Environ. Microbiol. 76, 1328-1333. doi: 10.1128/AEM.01042-09

Rosche, T. M., Yano, Y., and Oliver, J. D. (2005). A rapid and simple PCR analysis indicates there are two subgroups of Vibrio vulnificus which correlate with clinical or environmental isolation. Microbiol. Immunol. 49, 381-389. doi: 10.1111/j.1348-0421.2005.tb03731.x

Sanjuán, E., and Amaro, C. (2004). Protocol for specific isolation of virulent strains of Vibrio vulnificus serovar E (biotype 2) from environmental samples. Appl. Environ. Microbiol. 70, 7024-7032. doi: 10.1128/AEM.70.12.7024-7032.2004

Sanjuán, E., González-Candelas, F., and Amaro, C. (2011). Polyphyletic origin of Vibrio vulnificusbiotype 2 as revealed by sequence-based analysis. Appl. Environ. Microbiol. 77, 688-695. doi: 10.1128/AEM.01263-10

Satchell, K. J. F. (2015). Multifunctional-autoprocessing repeats-intoxin (MARTX) Toxins of Vibrios. Microbiol. Spectr. 3:VE-0002-2014. doi: 10.1128/microbiolspec.VE-0002-2014

Schmidt, H. A., Strimmer, K., Vingron, M., and von Haeseler, A. (2002). TREE-PUZZLE: maximum likelihood phylogenetic analysis using quartets and parallel computing. Bioinformatics 18, 502-504. doi: 10.1093/bioinformatics/ 18.3.502

Segerman, B. (2012). The genetic integrity of bacterial species: the core genome and the accessory genome, two different stories. Front. Cell. Infect. Microbiol. 2:116. doi: $10.3389 /$ fcimb. 2012.00116
Shimodaira, H. (2002). An approximately unbiased test of phylogenetic tree selection. System. Biol. 51, 492-508. doi: 10.1080/106351502900 69913

Shimodaira, H., and Hasegawa, M. (1999). Multiple comparisons of log-likelihoods with applications to phylogenetic inference. Mol. Biol. Evol. 16, 1114-1116. doi: 10.1093/oxfordjournals.molbev.a026201

Strimmer, K., and Rambaut, A. (2002). Inferring confidence sets of possibly misspecified gene trees. Proc. R. Soc. B Biol. Sci. 269, 137-142. doi: $10.1098 /$ rspb.2001.1862

Tatusov, R. L. (2000). The COG database: a tool for genome-scale analysis of protein functions and evolution. Nucleic Acids Res. 28, 33-36. doi: $10.1093 / \mathrm{nar} / 28.1 .33$

Tavaré, S. (1986). Some probabilistic and statistical problems in the analysis of DNA sequences. Am. Math. Soc. Lect. Math. Life Sci. 17, 57-86.

Tison, D. L., Nishibuchi, M., Greenwood, J. D., and Seidler, R. J. (1982). Vibrio vulnificus biogroup 2: new biogroup pathogenic for eels. Appl. Environ. Microbiol. 44, 640-646.

Wang, H., Ooi, B. C., Tan, K. L., Ong, T. H., and Zhou, L. (2003). BLAST++: BLASTing queries in batches. Bioinformatics 19, 2323-2324. doi: 10.1093/bioinformatics/btg310

Wolf, Y. I., Snir, S., and Koonin, E. V. (2013). Stability along with extreme variability in core genome evolution. Genome Biol. Evol. 5, 1393-1402. doi: 10.1093/gbe/evt098

Xu, Q., Dziejman, M., and Mekalanos, J. J. (2003). Determination of the transcriptome of Vibrio cholerae during intraintestinal growth and midexponential phase in vitro. Proc. Natl. Acad. Sci. U.S.A. 100, 1286-1291. doi: $10.1073 /$ pnas. 0337479100

Yu-Chung, C., Ming-Chung, C., Yin-Ching, C., and Chii-Ling, J. (2004). Characterization and virulence of hemolysin III from Vibrio vulnificus. Curr. Microbiol. 49, 175. doi: 10.1007/s00284-004-4288-5

Conflict of Interest Statement: The authors declare that the research was conducted in the absence of any commercial or financial relationships that could be construed as a potential conflict of interest.

Copyright @ 2018 Roig, González-Candelas, Sanjuán, Fouz, Feil, Llorens, BakerAustin, Oliver, Danin-Poleg, Gibas, Kashi, Gulig, Morrison and Amaro. This is an open-access article distributed under the terms of the Creative Commons Attribution License (CC BY). The use, distribution or reproduction in other forums is permitted, provided the original author(s) or licensor are credited and that the original publication in this journal is cited, in accordance with accepted academic practice. No use, distribution or reproduction is permitted which does not comply with these terms. 